\title{
Saturation of SERCA's lipid annulus may protect against its thermal inactivation
}

Val A. Fajardo ${ }^{1,2,3}$, Natalie Trojanowski ${ }^{2,3}$, Laura M. Castelli ${ }^{2,4}$, Paula M. Miotto ${ }^{2,4}$, Foyinsola Amoye ${ }^{2,4}$, Wendy E. Ward ${ }^{2,3,4}$, A. Russell Tupling ${ }^{1}$, Paul J. LeBlanc ${ }^{2,3 *}$

${ }^{1}$ Department of Kinesiology, University of Waterloo, Waterloo, ON, Canada ${ }^{2}$ Center for Bone and Muscle Health, Brock University, St. Catharines, ON, Canada ${ }^{3}$ Department of Health Sciences, Brock University, St. Catharines, ON, Canada ${ }^{4}$ Department of Kinesiology, Brock University, St. Catharines, ON, Canada

Total word count: 4,399

*Corresponding author:

Paul J. LeBlanc, PhD

Department of Health Sciences

Brock University

1812 Sir Isaac Brock Way

St. Catharines, ON

L2S 3A1 


\begin{abstract}
The sarco(endo)plasmic reticulum $\mathrm{Ca}^{2+}$-ATPase (SERCA) pumps are integral membrane proteins that catalyze the active transport of $\mathrm{Ca}^{2+}$ into the sarcoplasmic reticulum, thereby eliciting muscle relaxation. SERCA pumps are highly susceptible to oxidative damage, and cytoprotection of SERCA dampens thermal inactivation and is a viable therapeutic strategy in combating diseases where SERCA activity is impaired, such as muscular dystrophy. Here, we sought to determine whether increasing the percent of saturated fatty acids (SFA) within SERCA's lipid annulus through diet could protect SERCA pumps from thermal inactivation. Female Wistar rats were fed either a semi-purified control diet (AIN93G, 7\% soybean oil by weight) or a modified AIN93G diet containing high SFA (20\% lard by weight) for 17 weeks. Soleus muscles were extracted and SERCA lipid annulus and activity under thermal stress were analyzed. Our results show that SERCA's lipid annulus is abundant with short-chain (12-14 carbon) fatty acids, which corresponds well with SERCA's predicted bilayer thickness of $21 \AA$. Under control-fed conditions, SERCA's lipid annulus was already highly saturated (79\%), and high-fat feeding did not increase this any further. High-fat feeding did not mitigate the reductions in SERCA activity seen with thermal stress; however, correlational analyses revealed significant and strong associations between \% SFA and thermal stability of SERCA activity with greater \%SFA being associated with lower thermal inactivation and greater $\%$ polyunsaturation and unsaturation index being associated with increased thermal inactivation. Altogether, these findings show that SERCA's lipid annulus may influence its susceptibility to oxidative damage, which could have implications in muscular dystrophy and age-related muscle wasting.
\end{abstract}

Keywords: high fat diet, saturated fats, monounsaturated fats, polyunsaturated fats, heat stress 


\section{Highlights (85 characters each with spaces)}

- SERCA's lipid annulus in rat soleus was measured after immunoconcentration.

- Short fatty acid chains surround SERCA and may ensure optimal hydrophobic matching.

- SERCA's annulus is highly saturated in control-fed and high-fat fed rats.

- Greater saturation strongly associates with small levels of thermal inactivation.

- Greater unsaturation strongly associates with large levels of thermal inactivation.

Abbreviations: $\left[\mathrm{Ca}^{2+}\right]_{\mathrm{i}}$, intracellular $\mathrm{Ca}^{2+}$; Hsp70, heat shock protein 70; MUFA, monounsaturated fatty acids; PUFA, polyunsaturated fatty acids; ROS, reactive oxygen species; SFA, saturated fatty acids; SR, sarcoplasmic reticulum; SERCA, sarco(endo)plasmic reticulum $\mathrm{Ca}^{2+}$-ATPase; UI, unsaturation index 


\section{Introduction}

The sarco(endo)plasmic reticulum $\mathrm{Ca}^{2+}$-ATPase (SERCA) pumps are integral membrane proteins that are critical in eliciting muscle relaxation by catalyzing the active transport of $\mathrm{Ca}^{2+}[1]$. In resting skeletal muscle, SERCA pumps act to maintain low intracellular $\mathrm{Ca}^{2+}\left[\mathrm{Ca}^{2+}\right]_{\mathrm{i}}$ and a 1:10000 gradient between the cytosol and the sarcoplasmic reticulum (SR) [2]. The maintenance of low $\left[\mathrm{Ca}^{2+}\right]_{\mathrm{i}}$ is critical for muscle health, as $\mathrm{Ca}^{2+}$ dysregulation is a common feature in many skeletal myopathies and can lead to inflammation, elevated reactive oxygen species (ROS) production, muscle proteolysis, and eventual cell death [3-8]. Muscular dystrophy is one of the most-studied myopathies demonstrating the importance of $\mathrm{Ca}^{2+}$ regulation, and studies have shown that SERCA function is severely impaired in dystrophic muscles [9] and improving SERCA function improves muscle morphology and function [10-13].

Structurally, SERCA contains several amino acid residues like cysteine, tyrosine, and lysine that act as oxidative-sensitive targets, and in conditions of high oxidative stress, SERCA function is impaired [14-18]. Protein-protein interactions between heat shock protein 70 (Hsp70) and SERCA has been shown to protect SERCA from oxidative damage that occurs under heat stress, thereby mitigating thermal inactivation [19]. These seminal findings led to studies demonstrating that Hsp70 overexpression, either through transgenic or pharmacological induction, improved SERCA function and skeletal muscle pathology in $m d x$ mice, a mouse model for Duchenne's muscular dystrophy [10]. Together these findings suggest that protection of SERCA from cytotoxic damage may be a viable therapeutic strategy against muscular dystrophy. 
Phospholipids are important components to biological membranes that, in general, are composed of two hydrophobic fatty acid tails and one hydrophilic phosphate head group attached to a glycerol backbone. Fatty acids, especially highly unsaturated fatty acids that contain multiple double bonds, are susceptible to lipid peroxidation, which leads to the production of peroxyl fatty acid radicals and propagation of oxidative stress [20-22]. Given that the SERCA pumps are integral membrane proteins that are surrounded by membrane phospholipids, increased lipid peroxidation may negatively influence SERCA's physical structure and function. Thus, we sought to determine whether the lipid composition of naturally occurring lipid annulus around SERCA influences SERCA's susceptibility to oxidative damage. Specifically, we examined SERCA's phospholipid fatty acid annulus in relation to thermal inactivation in soleus muscles from female rats fed either a standard control diet or a high-fat diet (HFD) enriched with saturated fats. We hypothesized that consumption of the HFD would increase the percent of saturated fatty acids (SFA) within SERCA's lipid annulus and would protect SERCA from heat stress.

\section{Materials and Methods}

\section{Animals and Diet}

We used female Wistar rat dams that were part of a larger study examining the influence of maternal diet on offspring musculoskeletal health [23]. Dams were housed at $22 \pm 1{ }^{\circ} \mathrm{C}$ with a $12: 12 \mathrm{~h}$ light-dark cycle and fed either a control diet (control, AIN93G containing 7\% soybean oil by weight, TD.94045, Harlan Teklad, Mississauga, ON, Canada) or a high-fat diet (modified AIN93G containing 20\% lard by weight, TD.02016, 
Harlan Teklad) ad libitum for a total of 17 weeks, which included 1 week of breeding, 3 weeks of gestation, and 3 weeks of lactation. By design, body weight of dams fed HFD was higher post-lactation and complete body weight data are reported elsewhere [23]. Fatty acid analyses of the control and HFD diets are presented in Table 1 to highlight the dominant fatty acids that were expected to be reflected in SERCA's lipid annulus. The control diet is characterized by $18 \%$ SFA, $21 \%$ monounsaturated fatty acids (MUFA), and $60 \%$ polyunsaturated fatty acids (PUFA). The HFD, is characterized by $42 \%$ SFA, 40\% MUFA, and 18\% PUFA.

\section{Soleus collection and homogenization}

Dams were anesthetized with sodium pentobarbital in the intraperitoneal cavity (6mg/100g body weight). Soleus muscles were then extracted and homogenized with homogenizing buffer (250 mM sucrose, 5 mM HEPES, $10 \mathrm{mM} \mathrm{NaN}_{3}, \mathrm{pH} 7.5$ ) supplemented with a phosphatase inhibitor cocktail (PhosStop, Roche, Laval, QC, Canada), and a protease inhibitor cocktail (cOmplete Mini EDTA-free, Roche). Muscle homogenates were then stored at $-80^{\circ} \mathrm{C}$ until further analyses.

\section{Analyses of SERCA's lipid annulus}

To analyze SERCA's lipid annulus, SERCA2a was immunoconcentrated from soleus homogenates as previously described [24] using $40 \mu \mathrm{g}$ of SERCA2a antibody (2A7-A1, ab2861, Abcam, Cambridge, MA, USA) and $1.6 \mathrm{mg}$ of total protein from whole homogenates. Next, total lipids from the SERCA2a eluent were extracted according to Folch et al [25], and the total phospholipid pool was separated from neutral 
lipids using thin layer chromatography (60^̊, EMD, Mississauga, Ontario, Canada) with a hexane:diethylether:acetic acid (70:30:1) solvent system [26], which mobilizes neutral lipids but not phospholipids [27]. The immobile phospholipid pool was then methylated with $6 \% \mathrm{H}_{2} \mathrm{SO}_{4}$ in methanol overnight at $50^{\circ} \mathrm{C}$, and the fatty acid composition of the fatty acid methyl esters were analyzed by gas chromatography [28]. Fatty acids were identified by comparison of retention times with those of a known standard (Supelco 37 component FAME mix, Supelco, PA, USA), and absolute amounts (nmol) were calculated with the aid of the internal standard, tridecanoic acid (13:0), which was added to the samples immediately prior to the methylation process. The percent mol fraction of each fatty acid was calculated using the sum of the absolute amounts of the individual fatty acyl methyl esters as the denominator.

\section{SERCA activity and thermal stress}

Maximal SERCA activity assays during thermal stress were examined as previously described [19]. Briefly, soleus homogenates were incubated in a water bath set to $37^{\circ} \mathrm{C}$ for 0 (baseline), $30 \mathrm{~min}$, and $60 \mathrm{~min}$. Next, $\mathrm{Ca}^{2+}$-dependent ionophoresupported SERCA activity was assessed at each timepoint using an enzyme-linked spectrophotometric plate reader assay [29] with $\mathrm{Ca}^{2+}$ concentrations ranging from $p \mathrm{Ca}$ 7.0 - 5.0; and maximal SERCA activity was taken from the raw data.

\section{Statistics}

All values presented here are as means \pm standard error (SE). Differences between fatty acid composition in control and HFD were examined using a Student's t- 
test. A two-way repeated ANOVA was used to examine the effects of HFD on thermal inactivation of SERCA at baseline, and after $30 \mathrm{~min}$ and $60 \mathrm{~min}$ of heat stress. A Pearson's correlation was used to examine associations between \%SFA, \%MUFA, \%PUFA, and UI with changes in maximal SERCA activity after $60 \mathrm{~min}$ of heat stress. GraphPad Prism ${ }^{\mathrm{TM}}$ was used to perform all statistical tests and statistical significance was set to $p \leq 0.05$.

\section{Results and Discussion}

We examined the potential influence of SERCA's lipid annulus on thermal inactivation to determine whether increased \%SFA could protect SERCA from oxidative damage and minimize the effects of heat stress. To our knowledge, this is the first study examining specifically SERCA's lipid annulus after immunoconcentration, as most studies examining the influence of the lipid environment on SERCA function in skeletal muscle have relied on isolated SR membrane preparations [30-33]. We have found that SERCA's lipid annulus in female rat soleus muscles are comprised primarily of short chain fatty acids (12-14 carbons) which make up 50\% of the total phospholipid fatty acyl pool (Table 2). Considering that the bilayer thickness of SERCA has been proposed to be $21 \AA$ [34], which can be achieved with phospholipids containing 12-14 carbon long fatty acids [35], we propose that the abundance of the short fatty acid chains attached to SERCA helps to ensure optimal hydrophobic and hydrophilic matching.

In addition, our results show that SERCA's lipid annulus from rat soleus muscles are highly saturated with $79 \%$ SFA (Table 2). These results may be in contrast to what we have previously observed in purified SR membranes, where compared to red 
gastrocnemius and white gastrocnemius muscles, the SR membranes from rat soleus muscles were highly unsaturated [31]. However, without analyses of SERCA's lipid annulus across these muscle types it is difficult to make direct comparisons, and therefore, future studies are required. It would also be of interest to determine whether the lipid annulus pertaining to SERCA1a differs from that of SERCA2a, since we only examined SERCA2a in this study as rat soleus is dominated by type I fibres [36], which are abundant with SERCA2a [24].

In response to HFD, we found that SERCA's lipid annulus was generally nonresponsive. Although HFD contained significantly higher amounts of SFA (14:0, 16:0 and 18:0), there was no significant increase in these particular fatty acids in SERCA's lipid annulus nor was there an increase in \%SFA or a concomitant reduction in unsaturation index (UI, Table 2). In addition, although HFD contained approximately 40\% MUFA primarily from 18:1 (36\%, Table 1), this did not translate to alterations in 18:1 in SERCA's lipid annulus. This is in contrast to SR response to HFD, whereby rats fed high fish oil led to increased SR 20:5n3, 22:5n3, and 22:6n3 content, whereas high corn oil increased 18:2n6 content [37]. Further, we have recently shown that dietary supplementation of 22:6n3 for 8 weeks increased SR 22:6n 3 content in rat skeletal muscle [30]. It is possible that the lack of increase in percent saturation in response to HFD may be due to the already high amount of \%SFA found in SERCA's lipid annulus under control-fed conditions, and may also indicate that SERCA's lipid annulus is tightly regulated possibly to maintain optimal hydrophobic matching. Furthermore, skeletal muscle membrane fatty acid composition may be less responsive to SFA in the diet and more responsive to $n 3$ and $n 6$ PUFAs [38]. 
With the lack of dietary response, particularly no increase in \%SFA in the HFD group, it was not surprising that high-fat feeding in this study did not mitigate thermal inactivation of SERCA with similar \%reductions in maximal SERCA activity across control and HFD groups after 30 min and 60 min incubations (Figure 1). Nevertheless, correlational analyses within our dataset revealed significant and strong associations, with an increase in \%SFA being strongly associated with smaller absolute reductions in maximal SERCA activity after 60 min of heat stress (Figure 2A). Conversely, increased \%PUFA and UI were strongly associated with larger absolute reductions in maximal SERCA activity; and a similar direction of association was observed with \%MUFA; however, this was not significant $(p=0.15$, Figure $2 \mathrm{C}-\mathrm{D})$. Taken together, these data are consistent with our hypothesis and suggest that increasing \%SFA in SERCA's lipid annulus may protect SERCA from cytotoxic effects of oxidative damage, potentially by slowing the production of peroxyl fatty acid radicals and the propagation of oxidative stress. On the other hand, an increase in \%PUFA and UI may increase SERCA's susceptibility to oxidative damage given the presence of multiple double bonds, which are more susceptible to lipid peroxidation. Although future studies designed specifically to alter SERCAs lipid annulus either in rodent or cellular models will aid in substantiating these claims, the results presented here provide the first indication as to the influence of SERCA's lipid annulus on mediating SERCA's susceptibility to oxidative damage.

Our findings may have implications in the development of therapeutic strategies to combat Duchenne's muscular dystrophy, a severe form of muscular dystrophy with currently no cure [39]. An increase in ROS production is a well known characteristic 
pertaining to dystrophic muscles [40], and thus protecting SERCA function from

oxidative damage, in order to better regulate $\left[\mathrm{Ca}^{2+}\right]_{i}$, represents an attractive therapeutic strategy. This has been demonstrated by increasing protein-protein interactions with Hsp70 and SERCA, which offers SERCA cytotoxic protection in the face of oxidative stress and ultimately mitigates muscular dystrophy [10, 19, 41]. In this respect, our results demonstrating that certain lipid-protein interactions, particularly SFA-SERCA, can dampen the effects of thermal inactivation perhaps provide another avenue to protect SERCA from oxidative damage in muscular dystrophy. Future studies should examine: the potential changes in SERCA's lipid annulus in dystrophic muscles; whether these changes may predispose SERCA to oxidative damage; and whether increasing \%SFA in SERCA's lipid annulus in dystrophic muscle is a viable therapeutic option in improving muscle health. In addition to muscular dystrophy, future studies should examine the effect of SFA in SERCA's lipid annulus in other conditions characterized by increased ROS production such as age-related muscle wasting [42].

In summary, our findings provide the first evidence suggesting that SERCA's lipid annulus may influence its susceptibility to oxidative stress. Although, SERCA's lipid annulus was not responsive to the HFD employed in this study, several factors may account for this, including the design of the HFD, which was formulated to mimic an unhealthy diet in human populations, rather than a diet with supraphysiological levels of fat. Despite the lack in dietary response, correlational analyses within our dataset demonstrate strong associations between greater \%SFA and reduced thermal inactivation and greater \%PUFA and UI and increased thermal inactivation. We anticipate that these 
findings will have implications in conditions of muscle disease (ie. muscular dystrophy) and aging where an increase in ROS is a common feature. 


\section{Acknowledgements}

Funding: Analyses supported by a Discovery Grant from the Natural Sciences and Engineering Research Council of Canada to PJL. The in vivo portion of the study was funded by start-up funding from Brock University to WEW. VAF was supported through a Canadian Institutes of Health Research (CIHR) Doctoral Award; NT and PM were both supported through CIHR Master's Awards; LC was supported through an Ontario Graduate Scholarship. WEW holds a Canada Research Chair in Bone and Muscle Development. 


\section{References}

[1] A.R. Tupling, The sarcoplasmic reticulum in muscle fatigue and disease: role of the sarco(endo)plasmic reticulum Ca2+-ATPase, Can J Appl Physiol, 29 (2004) 308-329.

[2] D. Gamu, E. Bombardier, I.C. Smith, V.A. Fajardo, A.R. Tupling, Sarcolipin provides a novel muscle-based mechanism for adaptive thermogenesis, Exercise and sport sciences reviews, 42 (2014) 136-142.

[3] F. Altamirano, J.R. Lopez, C. Henriquez, T. Molinski, P.D. Allen, E. Jaimovich, Increased resting intracellular calcium modulates NF-kappaB-dependent inducible nitricoxide synthase gene expression in dystrophic mdx skeletal myotubes, J Biol Chem, 287 (2012) 20876-20887.

[4] P.S. Brookes, Y. Yoon, J.L. Robotham, M.W. Anders, S.S. Sheu, Calcium, ATP, and ROS: a mitochondrial love-hate triangle, Am J Physiol Cell Physiol, 287 (2004) C817833.

[5] A.R. Burr, D.P. Millay, S.A. Goonasekera, K.H. Park, M.A. Sargent, J. Collins, F. Altamirano, K.D. Philipson, P.D. Allen, J. Ma, J.R. Lopez, J.D. Molkentin, Na+ dysregulation coupled with $\mathrm{Ca} 2+$ entry through $\mathrm{NCX} 1$ promotes muscular dystrophy in mice, Mol Cell Biol, 34 (2014) 1991-2002.

[6] P. Gailly, New aspects of calcium signaling in skeletal muscle cells: implications in Duchenne muscular dystrophy, Biochim Biophys Acta, 1600 (2002) 38-44.

[7] A. Gorlach, K. Bertram, S. Hudecova, O. Krizanova, Calcium and ROS: A mutual interplay, Redox biology, 6 (2015) 260-271.

[8] M.J. Spencer, D.E. Croall, J.G. Tidball, Calpains are activated in necrotic fibers from mdx dystrophic mice, J Biol Chem, 270 (1995) 10909-10914.

[9] J.S. Schneider, M. Shanmugam, J.P. Gonzalez, H. Lopez, R. Gordan, D. Fraidenraich, G.J. Babu, Increased sarcolipin expression and decreased sarco(endo)plasmic reticulum $\mathrm{Ca} 2+$ uptake in skeletal muscles of mouse models of Duchenne muscular dystrophy, $\mathrm{J}$ Muscle Res Cell Motil, 34 (2013) 349-356.

[10] S.M. Gehrig, C. van der Poel, T.A. Sayer, J.D. Schertzer, D.C. Henstridge, J.E. Church, S. Lamon, A.P. Russell, K.E. Davies, M.A. Febbraio, G.S. Lynch, Hsp72 preserves muscle function and slows progression of severe muscular dystrophy, Nature, 484 (2012) 394-398.

[11] S.A. Goonasekera, C.K. Lam, D.P. Millay, M.A. Sargent, R.J. Hajjar, E.G. Kranias, J.D. Molkentin, Mitigation of muscular dystrophy in mice by SERCA overexpression in skeletal muscle, J Clin Invest, 121 (2011) 1044-1052.

[12] D.A. Mazala, S.J. Pratt, D. Chen, J.D. Molkentin, R.M. Lovering, E.R. Chin, SERCA1 overexpression minimizes skeletal muscle damage in dystrophic mouse models, Am J Physiol Cell Physiol, 308 (2015) C699-709.

[13] K.J. Morine, M.M. Sleeper, E.R. Barton, H.L. Sweeney, Overexpression of SERCA1a in the mdx diaphragm reduces susceptibility to contraction-induced damage, Hum Gene Ther, 21 (2010) 1735-1739.

[14] F. Qin, D.A. Siwik, S. Lancel, J. Zhang, G.M. Kuster, I. Luptak, L. Wang, X. Tong, Y.J. Kang, R.A. Cohen, W.S. Colucci, Hydrogen peroxide-mediated SERCA cysteine 674 oxidation contributes to impaired cardiac myocyte relaxation in senescent mouse heart, Journal of the American Heart Association, 2 (2013) e000184. 
[15] S. Lancel, F. Qin, S.L. Lennon, J. Zhang, X. Tong, M.J. Mazzini, Y.J. Kang, D.A. Siwik, R.A. Cohen, W.S. Colucci, Oxidative posttranslational modifications mediate decreased SERCA activity and myocyte dysfunction in Galphaq-overexpressing mice, Circ Res, 107 (2010) 228-232.

[16] R.I. Viner, T.D. Williams, C. Schoneich, Peroxynitrite modification of protein thiols: oxidation, nitrosylation, and S-glutathiolation of functionally important cysteine residue(s) in the sarcoplasmic reticulum Ca-ATPase, Biochemistry, 38 (1999) 1240812415.

[17] A.R. Tupling, C. Vigna, R.J. Ford, S.C. Tsuchiya, D.A. Graham, S.G. Denniss, J.W. Rush, Effects of buthionine sulfoximine treatment on diaphragm contractility and SR Ca2+ pump function in rats, J Appl Physiol (1985), 103 (2007) 1921-1928.

[18] R.I. Viner, D.A. Ferrington, A.F. Huhmer, D.J. Bigelow, C. Schoneich, Accumulation of nitrotyrosine on the SERCA2a isoform of SR Ca-ATPase of rat skeletal muscle during aging: a peroxynitrite-mediated process?, FEBS Lett, 379 (1996) 286-290. [19] A.R. Tupling, A.O. Gramolini, T.A. Duhamel, H. Kondo, M. Asahi, S.C. Tsuchiya, M.J. Borrelli, J.R. Lepock, K. Otsu, M. Hori, D.H. MacLennan, H.J. Green, HSP70 binds to the fast-twitch skeletal muscle sarco(endo)plasmic reticulum Ca2+-ATPase (SERCA1a) and prevents thermal inactivation, J Biol Chem, 279 (2004) 52382-52389. [20] A. Ayala, M.F. Munoz, S. Arguelles, Lipid peroxidation: production, metabolism, and signaling mechanisms of malondialdehyde and 4-hydroxy-2-nonenal, Oxid Med Cell Longev, 2014 (2014) 360438.

[21] C. Mylonas, D. Kouretas, Lipid peroxidation and tissue damage, In Vivo, 13 (1999) 295-309.

[22] A.J. Hulbert, N. Turner, L.H. Storlien, P.L. Else, Dietary fats and membrane function: implications for metabolism and disease, Biol Rev Camb Philos Soc, 80 (2005) 155-169.

[23] P.M. Miotto, L.M. Castelli, F. Amoye, P.J. LeBlanc, S.J. Peters, B.D. Roy, W.E. Ward, Maternal high fat feeding does not have long-lasting effects on body composition and bone health in female and male Wistar rat offspring at young adulthood, Molecules, 18 (2013) 15094-15109.

[24] V.A. Fajardo, E. Bombardier, C. Vigna, T. Devji, D. Bloemberg, D. Gamu, A.O. Gramolini, J. Quadrilatero, A.R. Tupling, Co-Expression of SERCA Isoforms, Phospholamban and Sarcolipin in Human Skeletal Muscle Fibers, PLoS One, 8 (2013) e84304.

[25] J. Folch, M. Lees, G.H. Sloane Stanley, A simple method for the isolation and purification of total lipides from animal tissues, J Biol Chem, 226 (1957) 497-509.

[26] I.R. Kupke, S. Zeugner, Quantitative high-performance thin-layer chromatography of lipids in plasma and liver homogenates after direct application of 0.5 -microliter samples to the silica-gel layer, J Chromatogr, 146 (1978) 261-271.

[27] V.A. Fajardo, L. McMeekin, A. Basic, G.D. Lamb, R.M. Murphy, P.J. LeBlanc, Isolation of sarcolemmal plasma membranes by mechanically skinning rat skeletal muscle fibers for phospholipid analysis, Lipids, 48 (2013) 421-430.

[28] N.S. Bradley, G.J. Heigenhauser, B.D. Roy, E.M. Staples, J.G. Inglis, P.J. LeBlanc, S.J. Peters, The acute effects of differential dietary fatty acids on human skeletal muscle pyruvate dehydrogenase activity, J Appl Physiol (1985), 104 (2008) 1-9. 
[29] T.A. Duhamel, H.J. Green, R.D. Stewart, K.P. Foley, I.C. Smith, J. Ouyang, Muscle metabolic, SR $\mathrm{Ca}(2+)$-cycling responses to prolonged cycling, with and without glucose supplementation, J Appl Physiol (1985), 103 (2007) 1986-1998.

[30] V.A. Fajardo, E. Bombardier, T. Irvine, A.H. Metherel, K.D. Stark, T. Duhamel, J.W. Rush, H.J. Green, A.R. Tupling, Dietary docosahexaenoic acid supplementation reduces SERCA Ca transport efficiency in rat skeletal muscle, Chemistry and physics of lipids, (2015).

[31] V.A. Fajardo, E. Bombardier, K. Tran, A.H. Metherel, T. Irvine, G.P. Holloway, H.J. Green, K.D. Stark, A. Russell Tupling, Sarcoplasmic Reticulum Phospholipid Fatty Acid Composition and Sarcolipin Content in Rat Skeletal Muscle, The Journal of membrane biology, 248 (2015) 1089-1096.

[32] K. Funai, I.J. Lodhi, L.D. Spears, L. Yin, H. Song, S. Klein, C.F. Semenkovich, Skeletal Muscle Phospholipid Metabolism Regulates Insulin Sensitivity and Contractile Function, Diabetes, 65 (2016) 358-370.

[33] K. Funai, H. Song, L. Yin, I.J. Lodhi, X. Wei, J. Yoshino, T. Coleman, C.F.

Semenkovich, Muscle lipogenesis balances insulin sensitivity and strength through calcium signaling, J Clin Invest, 123 (2013) 1229-1240.

[34] A.G. Lee, Lipid-protein interactions in biological membranes: a structural perspective, Biochim Biophys Acta, 1612 (2003) 1-40.

[35] B.A. Lewis, D.M. Engelman, Lipid bilayer thickness varies linearly with acyl chain length in fluid phosphatidylcholine vesicles, J Mol Biol, 166 (1983) 211-217.

[36] D. Bloemberg, J. Quadrilatero, Rapid determination of myosin heavy chain expression in rat, mouse, and human skeletal muscle using multicolor immunofluorescence analysis, PLoS One, 7 (2012) e35273.

[37] C.D. Stubbs, A.E. Kisielewski, Effect of increasing the level of omega-3 fatty acids on rat skeletal muscle sarcoplasmic reticulum, Lipids, 25 (1990) 553-558.

[38] S.K. Abbott, P.L. Else, A.J. Hulbert, Membrane fatty acid composition of rat skeletal muscle is most responsive to the balance of dietary n-3 and n-6 PUFA, The British journal of nutrition, 103 (2010) 522-529.

[39] A.E. Emery, The muscular dystrophies, Lancet, 359 (2002) 687-695.

[40] J.R. Terrill, H.G. Radley-Crabb, T. Iwasaki, F.A. Lemckert, P.G. Arthur, M.D.

Grounds, Oxidative stress and pathology in muscular dystrophies: focus on protein thiol oxidation and dysferlinopathies, The FEBS journal, 280 (2013) 4149-4164.

[41] M.H. Fu, A.R. Tupling, Protective effects of Hsp70 on the structure and function of SERCA2a expressed in HEK-293 cells during heat stress, Am J Physiol Heart Circ Physiol, 296 (2009) H1175-1183.

[42] S.J. Meng, L.J. Yu, Oxidative stress, molecular inflammation and sarcopenia, Int J

Mol Sci, 11 (2010) 1509-1526. 


\section{Figure Legends}

Figure 1. High-fat feeding does not mitigate thermal inactivation of SERCA in rat soleus muscles. Maximal SERCA activity was assessed at baseline ( $0 \mathrm{~min}), 30 \mathrm{~min}$, and 60 min after muscle homogenates were incubated at $37^{\circ} \mathrm{C}$, and are presented as a percent of baseline for control and high-fat diet (HFD) conditions. Two-way repeated ANOVA revealed a significant main effect of time $(p<0.0001)$, but no main effect of diet $(p=$ $0.86)$ or interaction $(p=0.82)$.

Figure 2. Correlation analyses between A) \%SFA, B) \% MUFA, C) \%PUFA, and D) UI with the change in maximal SERCA activity observed after 60 min of heat stress. $n=16$ with 8 per dietary group. SFA, saturated fatty acids; MUFA, monounsaturated fatty acids; PUFA, polyunsaturated fatty acids; UI, unsaturation index $=\Sigma m_{\mathrm{i}} \mathrm{X} n_{\mathrm{i}}$ where $m_{\mathrm{i}}$ is the mol percentage and $n_{\mathrm{i}}$ is the number of carbon-carbon double bonds of the fatty acid. 
